Ann. Biol. anim. Bioch. Biophys., I970, 10 (2), 309-312.

\title{
VARIATIONS DANS LA TENEUR EN ADN DE DEUX POPULATIONS DE SPERMATOZOÏDES DE TAUREAU SÉPARÉES PAR SÉDIMENTATION
}

\author{
C. ESNAULT ( $\left.{ }^{1}\right)$, R. ORTAVANT $\left({ }^{1}\right)$ et E. SCHILling $\left({ }^{2}\right)$ \\ avec la collaboration technique de J. C. Nicolle et Geneviève Radernacher \\ (1) Laboratoive de Physiologie de la Reproduction. \\ Centre de Recherches de Tours, 37 - Nouzilly \\ Institut national de la Recherche agronomique \\ (2) Max-Plank-Institut für Tierzucht und Tierernährung \\ Mariensee Trenthorst
}

Les spermatozoïdes de Taureau peuvent être séparés en plusieurs populations par sédimentation dans un milieu de viscosité et de poids spécifique déterminés (Schilling, 1965). Les fractions inférieures sont constituées de spermatozoïdes dits "lourds " et les fractions supérieures, de spermatozoüdes dits "légers". Des inséminations artificielles effectuées avec ces deux types de spermatozoïdes n'ont pas permis à Bhattacharya et al. (1966) de constater une modification du rapport des sexes des veaux obtenus; par contre, les essais réalisés par Schilling (1965, I966) puis par KNAACK $(1967,1968)$ ont abouti à une modification significative de ce rapport. On peut donc supposer que, dans ces deux derniers cas, la méthode de sédimentation utilisée a permis un enrichissement des fractions séparées, soit en spermatozoïdes porteurs du chromosome $\mathrm{X}$, soit en spermatozoïdes porteurs du chromosome Y. Schilling et al. (1967), puis KNaAck (1968) ont observé des différences de taille entre les spermatozoïdes des fractions "lourdes " et ccux des fractions "légères ". Il devrait donc être possible de mettre en évidence une différence faible, mais détectable dans la teneur en ADN entre les spermatozoïdes des deux fractions (BAKER et al., 1965) puisque chez les Bovins la différence de taille entre les chromosomes $\mathrm{X}$ et $\mathrm{Y}$ est très grande (Makino, 1944). C'est ce qui a été tenté dans ce travail par microspectrophotométrie.

\section{MATÉRIEI, ETT MÉTHODES}

Les essais ont été effectués au cours de deux séries d'expériences réalisées en 1967 et 1968 sur les éjaculats de 7 Taureaux. La séparation des différentes fractions (Io environ) a été effectuée selon la technique de Schillring (1965) et les fractions supérieures et inférieures ont été respectivement prélevées. Chacune de celles-ci contient 7 à 16 p. roo des spermatozoüdes de la population initiale. 
Après séparation, le dilueur a été éliminé par centrifugation et lavage ( 2 fois) à l'aide d'une solution de citrate de sodium à 2,9 p. ıoo. La morphologie des spermatozoïdes lavés a été étudiée sur frottis fixés à la flamme ou aux vapeurs de formol et colorés soit au giemsa, soit à l'hématoxyline ferrique, soit à l'érythrosine, soit selon la technique de Feulgen. Les dimensions des têtes des spermatozoïdes ont été déterminées à l'aide d'un micromètre oculaire à $\times=$ I $25^{\circ}$ ou d'un microscope à projection $(X=2090)$.

Les frottis pour les mesures d'absorption en lumière visible après coloration selon la technique de Feulgen ou en UV ont été préparés en double sur lames de verre ou sur lames de quartz et fixés aux vapeurs de formol pendant I 5 minutes. Les mesures d'absorption ont été effectuées sur le microspectrophotomètre UMSP I Zeiss par balayage du noyau entier et intégration des densités optiques (CASPERsson, CARLson et Svensson, 1954) selon les techniques déjà décrites (Bouters et al., 1967).

En outre, des mesures ont été effectuées à $280 \mathrm{~m} \mu$ pour examiner l'interférence des protéines nucléaires (BENEKE, I965; KIEFER, I965).

\section{RÉSULTATS ET DISCUSSION}

Les résultats concernant les mesures microspectrophotométriques sont rapportés dans le tableau $\mathbf{r}$.

Les teneurs moyennes en ADN-UV des spermatozoïdes des deux populations diffèrent significativement de 3,7 p. Ioo. Systématiquement, pour chacun des taureaux examinés (à l'exception d'un seul pour lequel les valeurs sont identiques), les teneurs en ADN-UV des spermatozoïdes

TABLEAU I

Teneurs moyennes \pm erreur type en $A D N$ Feulgen et $A D N-U V$ des spermatozoüdes des fractions lourdes et des fractions légères obtenues après sédimentation

\begin{tabular}{|c|c|c|}
\hline $\begin{array}{c}\text { Caractéristiques des } \\
\text { mesures }\end{array}$ & Fraction " lourde" & Fraction " légère " \\
\hline $\begin{array}{l}\text { ADN-Feulgen }(560 \mathrm{~m} \mu) \\
\text { ADN-UV }(265 \mathrm{~m} \mu) \ldots \\
\text { Extinction totale } \dot{\mathrm{a}} \\
\quad 280 \mathrm{~m} \mu \ldots \ldots \ldots\end{array}$ & $\begin{array}{l}6,02 \pm 0,082 \\
6,73 \pm 0,030 \quad(150) \\
4,11 \pm 0,026 \\
\end{array}$ & 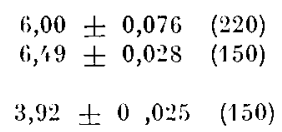 \\
\hline
\end{tabular}

( ) : nombre de spermatozoïdes mesurés.

des fractions "lourdes" sont plus élevées que celles des spermatozoïdes des fractions "légères ". Par contre, on n'observe aucune différence dans les teneurs moyennes en ADN-Feulgen : pour chacun des taureaux, les différences entre les teneurs en ADN-Feulgen des spermatozoïdes des deux fractions séparées par sédimentation sont tantôt dans un sens, tantôt dans l'autre.

Des exemples de discordance entre teneurs en ADN-UV et ADN-Feulgen des spermatozoïdes ont déjà été signalées (Bouters et al., 1967; EsNault et al., 1968) mais chaque fois on a constaté une constance des résultats obtenus pour l'ADN-UV comparée à la variabilité de ceux obtenus pour l'ADN-Feulgen. La variabilité observée dans ce dernier cas est supposée plus reliée à une modification de structure de l'ADN qu'à celle de la teneur des spermatozoïdes en ADN. Il est donc d'autant plus remarquable de constater ici une différence systématique de la teneur en ADN-UV 
entre les deux populations de spermatozoïdes. Les courbes de fréquence des teneurs en ADN-UV des spermatozoïdes individuels font apparaître qu'une partie au moins des spermatozoïdes de chacune des populations diffère d'une façon importante.

Les moyennes des intégrales des densités optiques mesurées à $280 \mathrm{~m} \mu$ sur les deux populations de spermatozoïdes montrent une différence de 4,9 p. roo. Ceci indique que les spermatozoïdes de la fraction "lourde " contiennent non seulement plus d'ADN mais aussi plus de protéines nucléaires que ceux de la fraction «légère ". Ceci peut être relié au fait que des déterminations d'acides aminés effectuées par l'un de nous montrent une teneur en arginine 4 à 8 p. Ioo plus élevée dans les spermatozoïdes de la fraction "lourde "(Schilling et Uzunov, 1970).

Enfin, de même que Schilling et al. (1967) et KNAACK (1968) l'avaient déjà trouvé, la taille moyenne des spermatozoïdes de la fraction " légère " est plus faible (tabl. 2). Cependant, la différence porte, dans ce travail, sur la largeur.

TABI,EAU 2

Taille $(\mu)$ des spermatozoides des fractions lourdes et des fractions légères obtenues après sédimentation

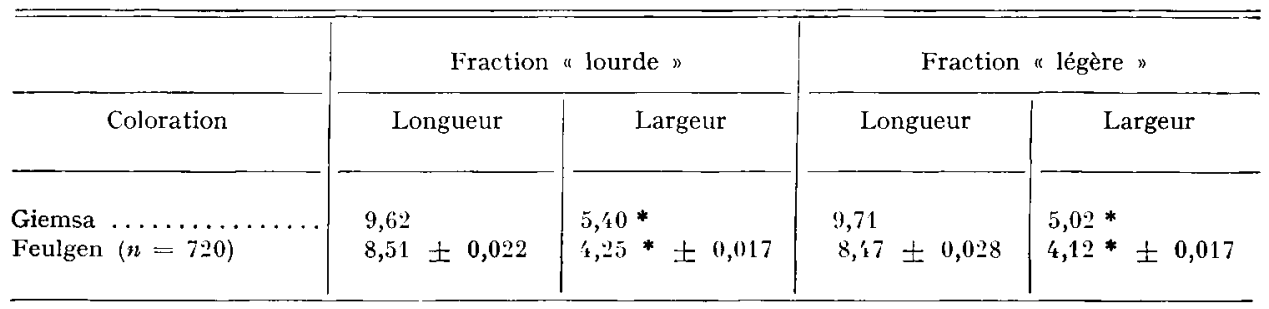

* Différence significative $\mathrm{P}<0,001$.

Ainsi, nous pouvons conclure que les teneurs en ADN et, vraisemblablement, en nucléoprotéines des spermatozoïdes des fractions "lourdes " et des fractions “ légères " séparées par sédimentation sont différentes. Il est possible que cette différence soit liée à la prédominance de spermatozoïdes $\mathrm{X}$ dans les premières et de spermatozoïdes $\mathrm{X}$ dans les autres car elle est de I'ordre de grandeur de celle calculée par LINDHAL (I960) et par BAKER et al. (1965). Mais cette hypothèse reste à démontrer : c'est ce que nous chercherons à faire au cours d'expériences futures.

Reçu pour publication en janvier 1970.

\section{SUMMARY}

\section{DIFFERENCES IN DNA CONTENTS OF TWO BULL, SPERM POPUI,ATIONS SEPARA'TED BY SEDIMENTATION}

Several populations of bull sperm were separated by sedimentation.

$\mathrm{U}$. V. microspectrophotometric $\left(26_{5} \mathrm{~m} \mu\right)$ measurements on the heaviest $v$. lightest fractions gave evidence for a $3.7 \mathrm{p}$. Ioo lower rate of UV-DNA in the lightest fraction. This difference is statistically significant. On the contrary, no difference was noticed in the average rates of Feulgen DNA. 
There was a 4.9 p. Ioo difference between the two sperm populations in the means of integrals of optical densities at $280 \mathrm{~m} \mu$.

The differences noticed acn possibly be due to the greater number of X-chromosome bearing spermatozoa in the lower fraction v. Y-chromosome bearing spermatozoa in the upper one.

\section{ZUSAMMENFASSUNG}

\section{UNTERSCHIEDE IN DEM DNS-GEHAIT VON ZWEI DURCH EIN SEDIMENTATIONSVERFAHREN GETRENNENTEN SPERMIENBESTÄNDEN VON BULLEN}

Durch ein Sedimentationsverfahren können Spermien von Bullen in schwere und leichte Formen getrennt werden. Schwere Spermien aus unteren Fraktionen weren durchwag länger und breiter und Befruchtungen mit ihnen führten zu einem höheren Anteil weiblicher Kälber, kleinere, leichtere Spermien zu mehr männlichen. In dieser Untersuchung wurde ermittelt, dass der UVDNS-Gehalt der schweren Spermien durchschnittlich 3,7\% höher war als für leichte Spermien. Die Messung der Feulgen-DNS erbrachte so eindeutige Ergebnisse nicht-die Werte für einzelne Bullen waren sehr unterschiedlich. Bei $280 \mathrm{~m} \mu$ betrug der Unterschied in der optischen Dichte $4,9 \%$ zugunsten der schweren Spermien. Dadurch wird aufgezeigt, dass schwere Spermien ausser einem höheren DNS-Gehalt auch mehr Nucleoproteide besitzen. Es ist möglich, dass die gemessenen Unterschiede zwischen beiden Spermienformen z. T. durch das Vorhandensein des X-oder $\mathrm{y}$-Chromosoms verursacht werden. Doch ist diese Hypothese in zukünftigen Untersuchungen exakt zu beweisen.

\section{RÉFÉRENCES BIBLIOGRAPHIQUES}

Baker F. N., Salisbury G. W., Fecheimer N. S., I965. Measurements of bovine chromosomes. Nature, 208, 97 .

BenEKE G., 1965. Extrapolationsverfahren zur Korrektur der UV-Absorptionkurven. Acta Histochem., Suppl. 6, 239 .

Bhattacharya $\quad$ B. C. H., Bangham A. D., Cro $\quad$ R. J., Keynes $\quad$ R. D., Rowson L. E. A., 1966. An attempt to predetermine the sex of calves by artificial insemination with spermatozoa separated by sedimentation. Nature, Lond., 211, 863

Bouters R., Esnault C., Salisbury G. W., Ortavant R., 1967. Discrepancies in analysis of deoxyribonucleic acid in rabbit spermatozoa, involving Feulgen staining (Feulgen DNA) and ultraviolet light absorption (UV-DNA) measurements. J. Reprod. Fert., 14, 355-363.

Caspersson T., Carló L., Svensson G., 1954. A scanning interference microscope arrangement. Exp. Cell Res., 7, 60I.

Esnault C., Orgebin-Crist M. C., Ortavant $\quad$ R. (cités par Orgebin-Crist M. C.) Ig69. Studies of the function of the epididymis. Biol. Reprod, Suppl.1, 155-175.

Kiefer G., 1965. Probleme bei der UV-photometrischen Bestimmung der Ribonukleinsäure in der Zelle. Acta Histochem., Suppl. 6, 255.

KNaAcK J., I968. Die willkürliche Geschlechtsbeeinflussung. Theorien, Hypothesen und Ergebnisse. Fortpfl. Haust, 4, 249-255.

KNAACK J., I968. Beitrag zum Spermiendimorphimus beim Rind. Fortpfl. Haust., 4, 256-269.

KNAACK J., 1968. Untersuchungen physiologischer Differenzen bei Sedimentierten Spermien. Fortpfl. Haust., 4, 270-278.

KNAACK J., I968. Willkürliche Geschlechtsbeeinflussung durch sedimentierte Rinderspermien (Ergebnisse eines Grossversuchs). Fortpfl. Haust., 4, 279-282.

Lindahl P. E., 1960. Experimental influence upon the distribution of the sexes in mammals by separation of male and female determining spermatozoa. $Z$. Tierz Zücht Biol., 74, 18I-197.

Makino S., 1944. Karyotypes of domestic cattle. Cytologia (Japan), 13, 247-264.

Schilling E., I965. Experimentelle Untersuchungen zur Geschlechtsbeeinflussung beim Rind. Naturwis., 53, 12.

Schilling E., I966. Experiments in sedimentation and centrifugation of bull spermatozoa and the sexratio. J. Reprod. Fert., 11, 469 .

Schilling E., Jazbec J., Schmid P., 1967. Grosse und Geschwindigkeit der Samenzellen vom Rind und Schaf und deren mögliche Beziehungen zum Geschlecht. Z. Tierz. Zücht Biol., 83, 331-339.

Schilling E., Uzunov G., I970. Stickstoffgehalt und Aminosäuren in schweren und leichten Samen zellen vom Rind und Schaf (Im Drück). 